\title{
Gout Treatment in the USA from 2009 to 2016: A Repeated Cross-sectional Analysis
}

J Gen Intern Med 36(4): 1134-6

DOI: $10.1007 / \mathrm{s} 11606-020-05942-8$

(C) Society of General Internal Medicine 2020

\section{BACKGROUND}

Approximately 8.3 million individuals (3.9\% of the US population) have gout, and its prevalence will continue to rise. ${ }^{1}$ We sought to characterize prescribing patterns and trends among office-based outpatient visits, in which gout was diagnosed.

\section{METHODS AND FINDINGS}

\section{Methods}

We used data from the 2009 to 2016 National Ambulatory Medical Care Survey (NAMCS), an annual cross-sectional survey of non-federally employed office-based physician visits. ${ }^{2}$ We identified visits by adults in which gout was coded using ICD-9 (274.XX) and ICD-10 (M10.XX) codes. For each visit, we categorized medications used to treat gout: (1) nonsteroidal anti-inflammatory agents (NSAIDs); (2) colchicine; (3) oral corticosteroids; and (4) urate-lowering therapy (ULT). Given the epidemic of opioid use in the USA and known use of opioids for acute pain, ${ }^{3}$ we also examined opioid prescriptions associated with these gout-related visits (see Table 1 for complete list of medications). We characterized treatment patterns by estimating the proportion of visits in which these medications were prescribed, along with patient and physician characteristics. Patient characteristics included age, gender, race/ethnicity, geographic region, insurance type, number of repeated visits, number of concomitant chronic conditions coded, and number of concomitant prescribed medications. Physicians were categorized by specialty as primary care (Internal Medicine and Family Medicine) versus specialties other than primary care.

We calculated change in the odds of visits being associated with a gout-related medication prescription across the 8-year period. We repeated analyses stratified by physician specialty. Lastly, we ran multivariable-adjusted logistic regression analysis to determine which demographic and clinical characteristics were associated with gout-related medication prescriptions.

Received February 17, 2020

Accepted May 4, 2020

Published online June 10, 2020
All analyses were performed using Stata 15.1 (StataCorp, College Station, TX), adjusted for the complex survey sampling design (e.g., unequal probability of selection, clustering, and stratification). ${ }^{4} \mathrm{~A} p$ value of 0.05 was used to test statistical significance.

\section{Findings}

Between 2009 and 2016, we identified 827 gout-related visits, representing approximately 3.4 million visits nationally. Overall, $74.8 \%$ of these visits had an associated prescription for gout-related therapy, including appropriate medications for gout treatment and opioids. The most common gout-related therapies prescribed were ULT $(45.1 \%)$, colchicine $(25.3 \%)$, and NSAIDs (22.5\%). From 2009 to 2016, opioid prescriptions increased, from 9.1 to $34.7 \%$ (odds ratio $[\mathrm{OR}]=1.73 ; 95 \% \mathrm{CI}=1.04$, $2.88)$, as did oral steroids, from 10.7 to $27.5 \%(\mathrm{OR}=1.48$; $95 \% \mathrm{CI}=1.04,2.08)$.

In multivariable-adjusted logistic regression analysis, men were more likely to be prescribed appropriate gout-related therapy than women $(\mathrm{OR}=2.72 ; 95 \% \mathrm{CI}=1.52-4.87, p=$ 0.005 ), as were patients with six or more concomitant prescription medications when compared to those with less than six medications $(\mathrm{OR}=5.11 ; 95 \% \mathrm{CI}=2.88-9.06$, $p<0.01)$. Most other patient and physician characteristics were not associated with gout-related medication prescription ( $p$ values $>0.05$; Table 2$)$.

\section{DISCUSSION}

In this repeated cross-sectional analysis of ambulatory visits to physicians, approximately one-quarter of visits among patients with gout did not include prescription for any goutrelated medication treatment. We observed several notable trends. First, prescriptions for ULT, the first-line preventive medication for gout, were the most commonly prescribed, but remained static over the latter half of the study period. Second, colchicine prescriptions decreased by $10 \%$ during years $2011-$ 2012 and increased steadily after 2012, perhaps due in part to the Food and Drug Administration's (FDA's) order to cease production of unapproved colchicine medications in 2010, leaving a single expensive drug on the market, followed by FDA approval of a generic version in 2012. ${ }^{5}$ Third, prescriptions for opioids and oral steroids significantly increased over the study period. Finally, patients with six or more 
Table 1 National Prescribing Trends for Gout-Related Medications and Opioids Among Adults with Gout in Office-Based Outpatient Care Visits in the USA, 2009-2016

\begin{tabular}{|c|c|c|c|c|c|c|c|c|}
\hline & \multicolumn{4}{|l|}{ Years $(\%)$} & \multirow[t]{2}{*}{ Overall } & \multicolumn{3}{|c|}{ Time trends } \\
\hline & 2009-2010 & 2011-2012 & 2013-2014 & 2015-2016 & & OR & $95 \% \mathrm{CI}$ & $p$ value \\
\hline $\begin{array}{l}\text { Visits in which any gout-related } \\
\text { medication prescribed }\end{array}$ & $76.3 \%$ & $69.4 \%$ & $72.7 \%$ & $80.1 \%$ & $74.8 \%$ & 1.08 & $0.76,1.53$ & 0.671 \\
\hline \multicolumn{9}{|l|}{ Stratified by medication group } \\
\hline Opioids* & $9.1 \%$ & $14.8 \%$ & $17.8 \%$ & $34.7 \%$ & $19.2 \%$ & 1.73 & $1.04,2.88$ & 0.034 \\
\hline Non-steroidal anti-inflammatory drugs ${ }^{\dagger}$ & $26.4 \%$ & $20.4 \%$ & $22.5 \%$ & $20.4 \%$ & $22.5 \%$ & 0.91 & $0.67,1.24$ & 0.552 \\
\hline Colchicine & $25.5 \%$ & $15.7 \%$ & $27.2 \%$ & $32.3 \%$ & $25.3 \%$ & 1.18 & $0.74,1.87$ & 0.485 \\
\hline Oral steroids ${ }^{\star}$ & $10.7 \%$ & $13.6 \%$ & $17.3 \%$ & $27.5 \%$ & $17.3 \%$ & 1.48 & $1.04,2.08$ & 0.028 \\
\hline Urate-lowering therapy ${ }^{\S}$ & $39.8 \%$ & $41.9 \%$ & $49.3 \%$ & $49.5 \%$ & $45.1 \%$ & 1.16 & $0.92,1.45$ & 0.204 \\
\hline Sample size & & & & & & Total & & \\
\hline Unweighted sample & 159 & 299 & 300 & 69 & - & \multicolumn{3}{|l|}{827} \\
\hline Weighted visits & 886,642 & 800,673 & 807,998 & 866,166 & - & \multicolumn{3}{|c|}{$3,361,479$} \\
\hline
\end{tabular}

Data are from National Ambulatory Medical Care Survey (NAMCS). *Includes codeine, meperidine, methadone, alfentanil, hydromorphone, morphine, oxycodone, pentazocine, propoxyphene, sufentanil, opium, levorphanol, oxymorphone, butorphanol, nalbuphine, buprenorphine, hydrocodone, dihydrocodeine, remifentanil, tapentadol, tramadol, and their combined products; 'Includes aspirin, diclofenac, ibuprofen, indomethacin, ketoprofen, ketorolac, naproxen, phenylbutazone, piroxicam, and tolmetin; Includes prednisone, methylprednisolone, dexamethasone, prednisolone, and betamethasone; ${ }^{\xi}$ Includes allopurinol, febuxostat, probenecid, lesinurad, and pegloticase

concomitant medications prescribed also had a higher likelihood of gout-related prescriptions, potentially increasing the risk of polypharmacy-related adverse events.

We note several limitations to this study. First, we were unable to account for concomitant diagnoses, and therefore, opioids and steroids may have been prescribed for indications other than gout. Second, NAMCS does not capture complete patient history, including symptom severity and previous therapy. Third, management of acute and chronic gout cannot be distinguished in the visit encounters, preventing more detailed understanding of prescription patterns. Finally, nonprescription NSAIDs are unaccounted for, likely underestimating NSAID exposure at all time points. Despite these limitations, our findings support the potential need for greater guideline-concordant gout management across all specialties. Understanding of the benefits of guideline-concordant gout management may reduce gout flares and improve patient outcomes.

Table 2 Multivariable-Adjusted Analysis of Factors Associated with Appropriate Gout-Related Medication Prescribing Among Adults with Gout in Office-Based Outpatient Care Visits in the USA, 2009-2016

\begin{tabular}{|c|c|c|c|}
\hline (Reference group in a parenthesis) & AOR & $95 \% \mathrm{CI}$ & $p$ value \\
\hline \multicolumn{4}{|l|}{ Age (45-64) } \\
\hline $18-44$ & 2.40 & $1.04,5.55$ & 0.07 \\
\hline$\geq 65$ & 0.63 & $0.35,1.16$ & 0.14 \\
\hline \multicolumn{4}{|l|}{ Gender (female) } \\
\hline Male & 2.72 & $1.52,4.87$ & 0.005 \\
\hline \multicolumn{4}{|l|}{ Race/ethnicity (non-Hispanic White) } \\
\hline Non-Hispanic Black & 0.70 & $0.35,1.38$ & 0.30 \\
\hline Hispanic & 0.60 & $0.20,1.77$ & 0.36 \\
\hline Other* & 1.49 & $0.65,3.39$ & 0.34 \\
\hline \multicolumn{4}{|l|}{ Region (Northeast) } \\
\hline Midwest & 1.17 & $0.56,2.48$ & 0.67 \\
\hline South & 0.56 & $0.26,1.20$ & 0.14 \\
\hline West & 0.70 & $0.35,1.40$ & 0.31 \\
\hline \multicolumn{4}{|l|}{ Primary source of payment (private) } \\
\hline Medicare & 1.92 & $0.98,3.79$ & 0.06 \\
\hline Medicaid & 1.56 & $0.38,6.43$ & 0.54 \\
\hline Other $^{\dagger}$ & 0.89 & $0.22,3.50$ & 0.86 \\
\hline \multicolumn{4}{|c|}{ Repeat of visits in the past 12 months (none) } \\
\hline $1-2$ visits & 1.61 & $0.76,3.43$ & 0.22 \\
\hline $3-5$ visits & 1.40 & $0.57,3.45$ & 0.47 \\
\hline $6+$ visits & 3.12 & $1.15,8.42$ & 0.03 \\
\hline \multicolumn{4}{|l|}{ Physician specialty (primary care) } \\
\hline Other & 1.44 & $0.74,2.82$ & 0.28 \\
\hline$\geq 4$ chronic conditions $(<4)$ & 0.69 & $0.36,1.33$ & 0.28 \\
\hline$\geq 6$ medications $(<6)$ & 5.11 & $2.88,9.06$ & 0.001 \\
\hline
\end{tabular}

Data are from National Ambulatory Medical Care Survey (NAMCS). AOR adjusted odds ratio. *Denotes Asian, American Indian and Alaska Native, Native Hawaiian, or Other Pacific Islander, or $\geq 2$ reported racial or ethnic groups; 'Denotes worker's compensation, self-pay, no charge, or other;

${ }^{7}$ Includes obstetrics and gynecology, cardiology, dermatology, urology, neurology, ophthalmology, otolaryngology, and other specialties 
Yu Pei Chock, $M D, M P H^{1}$

Joseph S. Ross, $M D, M H S^{2}$

Lisa G. Suter, $M D^{1,3}$

Taeho Greg Rhee, $\mathrm{PhD}^{4}$

${ }^{1}$ Section of Rheumatology, Department of Medicine, Yale University School of Medicine,

New Haven, CT, USA

${ }^{2}$ Section of General Medicine, Department of Internal Medicine, Yale School of Medicine,

New Haven, CT, USA

${ }^{3}$ Veterans Affairs Connecticut Health System,

West Haven, CT, USA

${ }^{4}$ Department of Public Health Sciences, School of Medicine, University of Connecticut,

Farmington, CT, USA

Corresponding Author: Yu Pei Chock, MD, MPH; Section of Rheumatology, Department of Medicine, Yale University School of Medicine New Haven, CT, USA (e-mail: yupeieugenia.chock@yale.edu).

Authors' Contribution Each author contributed substantially to this work in terms of conception, design, analysis, and writing. Each author reviewed and approved the final version of this manuscript.

\section{Compliance with Ethical Standards:}

Conflict of Interest (Representing the Past 36 Months): Dr. Ross declares the following interests: receiving research support through Yale University from Johnson and Johnson, the Food and Drug
Administration (FDA) (U01FD004585), Medical Devices Innovation Consortium (MDIC)/National Evaluation System for Health Technology (NEST), Centers of Medicare and Medicaid Services (CMS) (HHSM-50O2013-13018I), Agency for Healthcare Research and Quality (R01HS022882), National Heart, Lung and Blood Institute of the National Institutes of Health (NIH) (RO1HSO25164), and the Laura and John Arnold Foundation.

Dr. Suter declares that she co-chaired and served on the work group that developed the American College of Rheumatology's gout quality measures (volunteer position).

Drs. Chock and Rhee have no conflict of interests to disclose.

\section{REFERENCES}

1. Zhu Y. Prevalence of gout and hyperuricemia in the US general population: the national health and nutrition examination survey 2007-2008 prevalence of gout and hyperuricemia in the US. Arthritis Rheum. Invalid date:63(10):3136-3141.

2. NAMCS scope and sample design. https://www.cdc.gov/nchs/ahcd/ ahcd_scope.htm. Updated 2010. Accessed 18 July 2018.

3. Vijay A, Rhee TG, Ross JS. U.S. prescribing trends of fentanyl, opioids, and other pain medications in outpatient and emergency department visits from 2006 to 2015. Prev Med 2019;123:123-129.

4. Hsiao C. Understanding and using NAMCS and NHAMCS data. https:// www.cdc.gov/nchs/ppt/nchs2010/03_Hsiao.pdf. Updated 2010. Accessed 4 Aug 2018.

5. Kesselheim AS, Solomon DH. Incentives for drug development - the curious case of colchicine. N Engl J Med. Invalid date;362(22):2045-2047. https://doi.org/10.1056/NEJMp1003126.

Publisher's Note: Springer Nature remains neutral with regard to jurisdictional claims in published maps and institutional affiliations. 